\title{
Evidence for universal signatures of Zeeman-splitting-limited pseudogaps in superconducting electron- and hole-doped cuprates
}

\section{AUTHOR(S):}

Kawakami, T; Shibauchi, T; Terao, Y; Suzuki, M; Krusin-Elbaum, L

\section{CITATION:}

Kawakami, T ... [et al]. Evidence for universal signatures of Zeeman-splitting-limited pseudogaps in superconducting electron- and hole-doped cuprates. Physical Review Letters 2005, 95(1): 017001.

\section{ISSUE DATE:}

2005-07-01

URL:

http://hdl.handle.net/2433/39927

RIGHT:

Copyright 2005 American Physical Society 


\title{
Evidence for Universal Signatures of Zeeman-Splitting-Limited Pseudogaps in Superconducting Electron- and Hole-Doped Cuprates
}

\author{
Tsuyoshi Kawakami, ${ }^{1}$ Takasada Shibauchi, ${ }^{1, *}$ Yuhki Terao, ${ }^{1}$ Minoru Suzuki, ${ }^{1}$ and Lia Krusin-Elbaum ${ }^{2}$ \\ ${ }^{1}$ Department of Electronic Science and Engineering, Kyoto University, Kyoto 615-8510, Japan \\ ${ }^{2}$ IBM T. J. Watson Research Center, Yorktown Heights, New York 10598, USA
}

(Received 21 June 2004; published 29 June 2005)

\begin{abstract}
We probe the "normal" state in electron-doped (n-type) $\mathrm{Sm}_{2-x} \mathrm{Ce}_{x} \mathrm{CuO}_{4-\delta}$ through interlayer tunneling transport in magnetic fields up to $45 \mathrm{~T}$. The behavior of intrinsic high-field $c$-axis negative magnetoresistance (MR), which is accessed in small $30 \mathrm{~nm}$-high mesa structures, is characteristic of the pseudogap state. It follows a universal correlation between the excess low-energy dissipation due to the pseudogap and its closing field $H_{\mathrm{pg}}$ and is in close correspondence with the hole-doped ( $p$-type) $\mathrm{Bi}_{2} \mathrm{Sr}_{2} \mathrm{CaCu}_{2} \mathrm{O}_{8+y}$. The MR in the mesas and in the bulk crystals consistently gives a Zeeman relation between the pseudogap temperature $T^{*}$ and its closing field, pointing to a preeminent role of spin-singlet correlations in forming the pseudogap in cuprates, regardless of their $n$ or $p$ type.
\end{abstract}

DOI: 10.1103/PhysRevLett.95.017001

Superconductivity in cuprates can be induced by doping the parent antiferromagnetic insulator with either electrons or holes [1]. By many appearances, however, the phase diagram on the two sides of the doping divide seems to be particle-hole asymmetric. In addition to the lower transition temperatures $T_{c}$, in electron-doped (n-type) cuprates $\left(L_{2-x} \mathrm{Ce}_{x} \mathrm{CuO}_{4-\delta}\right.$, where $L=\mathrm{Nd}, \mathrm{Pr}, \mathrm{Eu}$, or Sm) the antiferromagnetic phase persists to much higher doping levels, while the superconductivity is observed in a narrower $(0.14 \lesssim x \leqq 0.18)$ range [2]. A feature that dominates large parts of the normal-state phase space is the anomalous depletion in the density of states (DOS) referred to as pseudogap. It is ubiquitous in the underdoped hole-doped ( $p$-type) cuprates [3], and has been also reported in underdoped albeit nonsuperconducting $\mathrm{Nd}_{2-x} \mathrm{Ce}_{x} \mathrm{CuO}_{4-\delta}$ (NCCO) $[4,5]$. The connection of this pseudogap to superconductivity is still not obvious, and, with experimental situation often contradictory [6-10], even its existence in the normal state of superconducting cuprates of $n$ type is far less certain. Absence or presence of a pseudogap in a superconducting electron-doped cuprate, if proved, may inform on two chief questions about high- $T_{c}$ : (a) are $n$ - and $p$-type cuprates fundamentally different, and/or (b) is the pseudogap phenomenon related to superconductivity at all?

A robust way to probe the pseudogap state is via interlayer transport $\rho_{c}$-a technique uniquely suitable at ultrahigh magnetic fields, as has been demonstrated in $p$-type $\mathrm{Bi}_{2} \mathrm{Sr}_{2} \mathrm{CaCu}_{2} \mathrm{O}_{8+y}$ (BSCCO) [11,12]. When the layered structure forms intrinsic tunnel junctions, $\rho_{c}$ is a good measure of the low-energy DOS. To expose the pseudogap state, first the long-range superconducting coherence must be destroyed, e.g., by applying appropriately high fields (conventionally, exceeding the upper critical field $H_{c 2}$ [13]). The recovery of the DOS by magnetic field to a fully ungapped state can be then sensitively detected by the negative interlayer magnetoresistance (MR). Indeed, we have established that in the hole-doped cuprates the pseu-
PACS numbers: 74.25.Dw, 74.25.Fy, 74.50.+r, 74.72.Jt

dogap can be closed by a high magnetic field [11] that scales with the pseudogap onset temperature $T^{*}$.

Here we report first interlayer tunneling transport experiments in $n$-type superconducting cuprates, fabricated (out of bulk crystals) as small $30 \mathrm{~nm}$-thin mesa structures consisting only of $\sim 50 \mathrm{CuO}_{2}$ planes comprising the tunnel junctions. The measurements on mesas were compared against the bulk behavior in fields up to $45 \mathrm{~T}$. The mesa structure was crucial in establishing the intrinsic behavior of the MR, for it circumvented the known crystal-growth problem of $n$-type cuprates where a secondary phase forms a quasi-two-dimensional long-range epitaxial structure parallel to the $\mathrm{CuO}_{2}$ sheets [14] - an inhomogeneity that could be masking the intrinsic physics. Our observations support a presence of the pseudogap that can be closed by a magnetic field in the laboratory range, and hence, for the first time not requiring any guesses or extrapolations.

We find that in one key aspect, the $p$ - and $n$-type superconducting cuprates are surprisingly similar: they follow an empirically "universal" correlation between the excess low-energy dissipation due to the pseudogap and its closing field $H_{\mathrm{pg}}$. A simple Zeeman scale connects the pseudogap temperature $T^{*}$ to $H_{\mathrm{pg}}$ in an $n$-type material, suggesting a common origin of the pseudogap irrespective of the charge carrier type - in both Zeeman splitting closing the pseudogap implies that the triplet spin excitation at high fields overcomes the spin-singlet pair correlations responsible for the gap in the spin spectrum and the orbital contribution is very small.

For this study we have chosen $\mathrm{Sm}_{2-x} \mathrm{Ce}_{x} \mathrm{CuO}_{4-\delta}$ (SCCO) crystals as having a distinct advantage for the interlayer transport: $\mathrm{Sm}$ ions show the antiferromagnetic transition at $T_{\mathrm{N}}=4.9 \mathrm{~K}$ [15] well below $T_{\mathrm{c}}$. This allows us to check for effects of the magnetic lanthanoid ions on the interlayer transport by comparing MR above and below $T_{\mathrm{N}}$. We used a self-flux method $[16,17]$ with the crucible turnover technique [18] to get platelike crystals with fluxfree and shiny surfaces, which is critical for the mesa 
fabrication. The as-grown crystals were annealed under $\mathrm{Ar}$ flow for $20 \mathrm{~h}$ at $950^{\circ} \mathrm{C}$. Ce concentration $x$ was determined by the energy dispersive $\mathrm{x}$-ray spectroscopy (EDS). Mesa structures with typical dimensions of $(8 \times 12) \mu \mathrm{m}^{2} \times$ $30 \mathrm{~nm}$ were fabricated using photolithography and ion milling techniques [19]. Au/Ag bilayer was evaporated on the "natural" surface of the annealed crystals, with two contacts on top of the mesa [Fig. 1(a)] enabling fourterminal measurements of the $c$-axis resistivity $\rho_{c}$ of the mesa alone. With the thickness of the secondary-phase layer in the range 4-8 $\mathrm{nm}$ and with the volume fraction of $1-3 \%$ [14], the height $(30 \mathrm{~nm})$ of our mesas is much smaller than typical separation between the neighboring secondary-phase layers. Eleven mesas measured showed consistently reproducible values of $\rho_{c}$, giving us confidence in null effect of the secondary phase. We note that in bulk crystals, the analysis of transport measurements $[4,20]$ may have been affected [21].

Figure 1 shows the temperature and field dependence of $\rho_{c}$ of an SCCO mesa sample with $x \approx 0.14 . \rho_{c}(T)$ exhibits metallic behavior over a wide temperature range [Fig. 1(b)]. At zero field, on cooling $\rho_{c}(T)$ articulates an upturn just above $T_{\mathrm{c}}$ followed by a sharp transition at $T_{\mathrm{c}} \sim$ $20 \mathrm{~K}$; the sharpness is a reflection of high homogeneity in the measured mesa region. The upturn becomes more pronounced when we apply magnetic field $H$ along the $c$ axis [Fig. 1(c)]. With increasing $H$, the transition is shifted to lower temperatures. At fixed temperatures $\rho_{c}$ on the upturn is systematically suppressed with $H$, and $\rho_{c}(T)$ follows a $\log T$ dependence. At high $T$, there is a small $H^{2}$ standard positive MR [22]; after subtracting $\alpha H^{2}$ term $\left(\alpha / \mu_{0}^{2}=2.6 \times 10^{-4} \Omega \mathrm{cm} / \mathrm{T}^{2}\right)$, all data collapse onto a single curve [inset in Fig. 1(c)]. This clearly uncovers negative MR below $\sim 38 \mathrm{~K}$, somewhat above the temperature of minimum $\rho_{c}$. These characteristic features, (i) the upturn, (ii) the shift of the maximum $\rho_{c}(T)$, (iii) the negative MR, and (iv) the $\log T$ behavior at low $T$, are all in close correspondence with the features observed in the interlayer transport in the pseudogap state of BSCCO $[11,13]$. We note that there is not much change in the intrinsic $\rho_{c}(T)$ at $T_{\mathrm{N}}$, which ensures that critical effects of $\mathrm{Sm}$ moments do not enter in our analysis.

The field dependence of $\rho_{c}$ in the mesa shown in Fig. 1(d) looks essentially the same as that in the $p$-type cuprates: $\rho_{c}$ is null up to the irreversibility field $H_{\text {irr }}$, and then it rises to a peak at $H_{\text {peak }}$, above which MR is negative. This negative MR is a natural consequence of a recovery of DOS depletion comprising the pseudogap [11,23] if the $c$-axis transport is via tunneling between $\mathrm{CuO}_{2}$ planes. In accordance with this, studies of $c$-axis current-voltage characteristics in an $n$-type cuprate [24] report behavior consistent with stacks of superconductor-insulatorsuperconductor junctions.

Let us now turn to the behavior of bulk SCCO crystals. The $c$-axis MR for a crystal $\left(1.0 \times 1.2 \times 0.025 \mathrm{~mm}^{3}\right)$ with $x \approx 0.14$ measured in a $45 \mathrm{~T}$ hybrid magnet [25] is shown
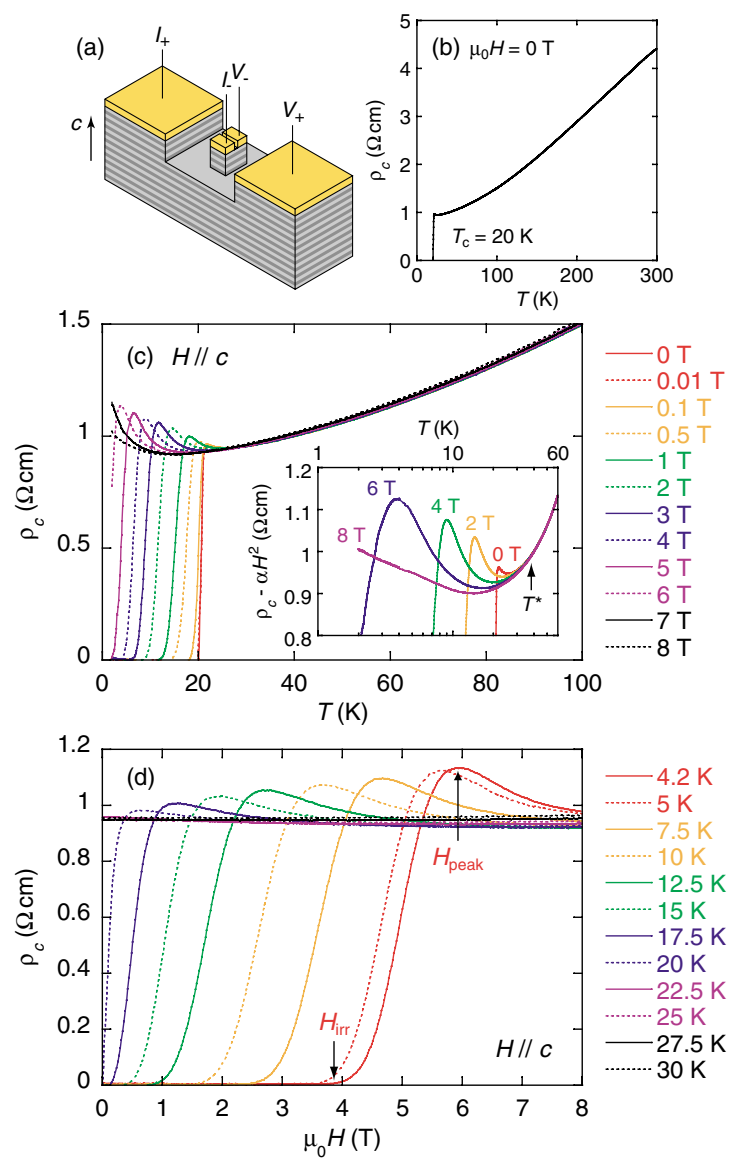

FIG. 1 (color online). (a) Schematic illustration of our mesa structure (not to scale). (b) Temperature dependence of $\rho_{c}$ in zero field for a mesa sample. (c) $\rho_{c}(T)$ curves in magnetic fields applied along the $c$ axis. A slight field spread of the curves at high $T$ is due to a small positive $\mathrm{MR} \propto H^{2}$, see text. After subtracting this MR all high- $T$ data follow a single curve (inset). Negative MR is observed below $T^{*} \approx 38 \mathrm{~K}$. (d) Field dependence of $\rho_{c}$ for the SCCO mesa sample.

in Fig. 2. In the bulk crystals, the $\rho_{c}$ values in comparison with mesas are higher and the upturn is enhanced [inset of Fig. 2(b)]. Some of this enhancement must originate from the chemical inhomogeneity we discussed earlier. However, the relative MR reveals behavior quantitatively consistent with the intrinsic mesa data. It shows a peak at $H_{\text {peak }}$, above which the MR is negative as shown in Fig. 2(a) for $H \| c$. At very high fields, the positive $\alpha H^{2}$ term becomes dominant with $\alpha\left(\alpha / \mu_{0}^{2}=2.2-2.5 \times\right.$ $10^{-4} \Omega \mathrm{cm} / \mathrm{T}^{2}$ ) nearly identical to that of the mesa. Furthermore, the "overshoot" of $\rho_{c}(H)$ near the peak, i.e., $\rho_{c}\left(H_{\text {peak }}\right)-\rho_{c}\left(H>H_{\text {peak }}\right)$, is roughly $0.15-0.2 \Omega \mathrm{cm}$ at low temperatures, which is comparable to that of the mesa. This is an important point: since the secondary phase is reported to have long-range epitaxial structure parallel to the $a b$ plane, $\rho_{c}$ in a bulk crystal may be expressed as a sum of the contributions from both phases. The consistency of the relative changes in mesa and bulk MRs suggests that the observed negative MR is of the same origin in both, 

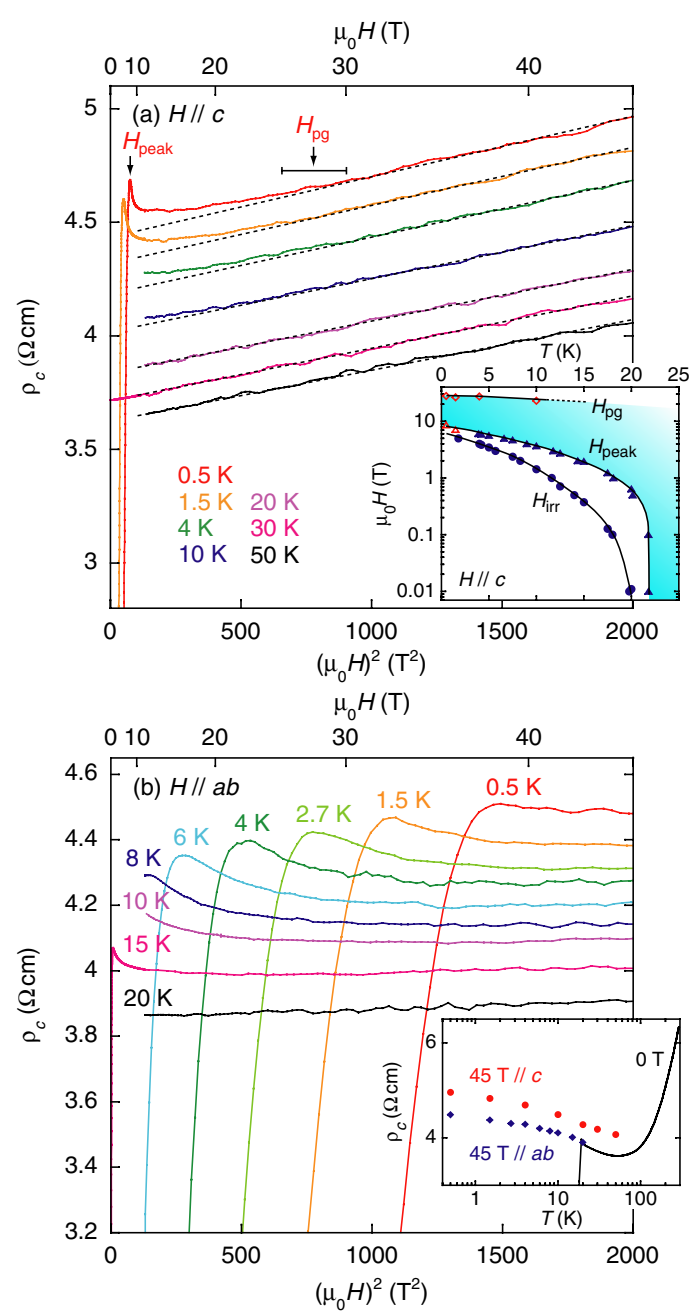

FIG. 2 (color online). $\rho_{c}$ vs $H^{2}$ at various temperatures in an SCCO bulk sample for (a) $H \| c$ and (b) $H \| a b$ [25]. Dashed lines are high-field fits to the $H^{2}$ dependence. Insets: (a) $H-T$ diagram for the mesa (closed symbols) and bulk (open symbols) samples. Lines are guides to the eye. Negative MR (shaded region) vanishes at $H_{\mathrm{pg}}(T)$. (b) $\rho_{c}(T)$ of the bulk sample without field (solid line) and in $45 \mathrm{~T}$ (filled symbols).

namely, it must come intrinsically from the field suppression of the pseudogap. We can write then the intrinsic $\rho_{c}$ in the normal state above $H_{\text {peak }}$ as

$$
\rho_{c}(T, H)=\rho_{c}^{\mathrm{n}}(T)+\Delta \rho_{c}(T, H)+\alpha H^{2},
$$

where $\rho_{c}^{\mathrm{n}}$ is ungapped resistivity in zero field, $\Delta \rho_{c}$ is the resistivity change arising from the pseudogap, and $\alpha$ is nearly $T$ independent at low $T$ [26]. The pseudogap closing field $H_{\mathrm{pg}}$-defined by $\Delta \rho_{c}\left(T, H_{\mathrm{pg}}\right)=0$-is determined to be 28( \pm 2$) \mathrm{T}$ for $H \| c$ (at $T \leq 4 \mathrm{~K})$. We note that here the field scales are such that for the first time the disappearance of negative MR can be observed without requiring further assumptions and extrapolations.

The temperature dependencies of $H_{\text {irr }}, H_{\text {peak }}$, and $H_{\mathrm{pg}}$ are plotted in the inset of Fig. 2(a). Consistently, the $H_{\text {peak }}(T)$ data for the mesa and the crystal follow a single curve. At low temperatures $(T \lesssim 10 \mathrm{~K}), H_{\mathrm{pg}}$ is nearly "flat" in temperature - a characteristic $T$ dependence also found in BSCCO [11,27].

A more precise determination of $T^{*} \approx 38( \pm 2) \mathrm{K}$ is obtained by plotting the temperature derivative of $\rho_{c}$; see Fig. 3. From this we obtain that $H_{\mathrm{pg}}$ and $T^{*}$ obey a simple Zeeman energy relation $k_{\mathrm{B}} T^{*} \approx g \mu_{\mathrm{B}} \mu_{0} H_{\mathrm{pg}}$ with $g \sim 2$ ( $k_{\mathrm{B}}$ is the Boltzmann constant and $\mu_{\mathrm{B}}$ is the Bohr magneton). Thus, the pseudogap energy scale found here lies on the same Zeeman line as for $p$-type BSCCO [11]; see inset in Fig. 4. The congruity between the $n$-type and $p$-type cuprates is further manifested through the uncovered empirical correlation [Fig. 4] between the observed maximum $\Delta \rho_{c}$ (a measure of the negative MR term and the lowenergy DOS) and the pseudogap energy scale:

$$
\Delta \rho_{c}^{\max } \propto H_{\mathrm{pg}}^{3} \quad \text { or } \quad \propto T^{* 3} .
$$

This correlation is robust, spanning 4 orders of magnitude difference in $\Delta \rho_{c}^{\max }$ between SCCO and BSCCO [11,23], and holds in other highly anisotropic cuprates on the two doping sides, such as NCCO $(x=0.15)$ [28] and La-doped $\mathrm{Bi}_{2} \mathrm{Sr}_{2} \mathrm{CuO}_{6+y}$ (BSCO) [29].

To test the relative contribution of orbital degrees in SCCO we applied field $H$ parallel to the $a b$ planes. As shown in Fig. 2(b), in this case the peak field is shifted to higher fields - an effect naturally explained by the strong superconducting anisotropy in layered cuprates [13,27]. At high fields, the positive MR term for $H \| a b$ is smaller than for $H \| c$ [30], and the negative part dominates. We observe negative MR irrespective of the field direction, which does rule out a two-dimensional localization as its origin. Moreover, $\rho_{c}(T)$ in the two field configurations at $45 \mathrm{~T}$

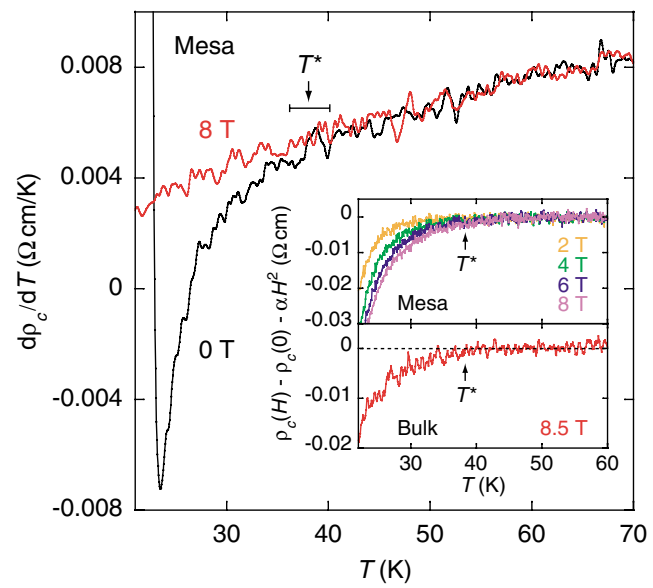

FIG. 3 (color online). $\quad \mathrm{d} \rho_{c} / \mathrm{d} T$ vs $T$ at 0 and $8 \mathrm{~T}$ in the mesa. The exposed downward departure of the zero-field $\mathrm{d} \rho_{c} / \mathrm{d} T$ from the high-field data highlights the $\rho_{c}(T)$ upturn due to DOS depletion below $T^{*}$. Inset: Temperature dependence of the negative MR term in the mesa and the bulk samples. All the data consistently indicate that the onset temperature $T^{*}$ is $38( \pm 2) \mathrm{K}$ (arrows) both in the mesa and the bulk-a witness to the intrinsic nature of the relevant features. 


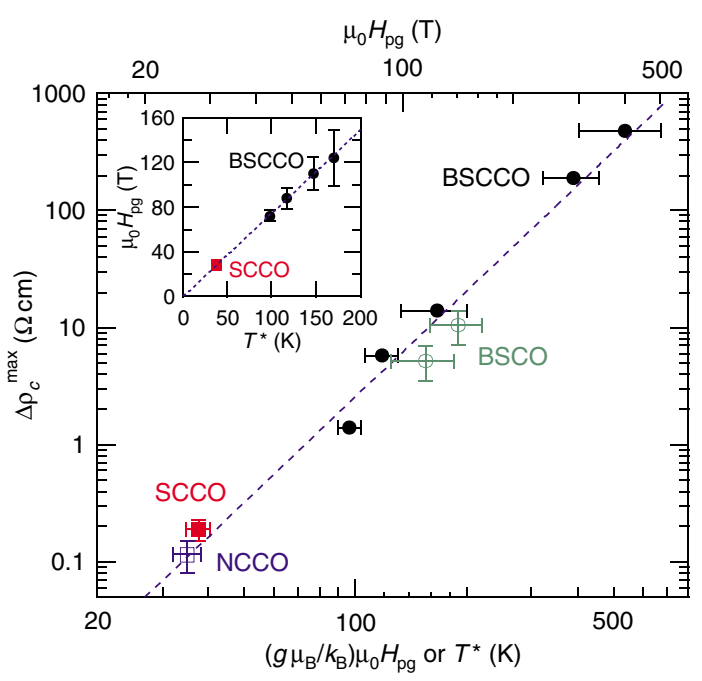

FIG. 4 (color online). Maximum $\Delta \rho_{c}$ in SCCO (solid squares) and BSCCO (solid circles) as a function of obtained $H_{\mathrm{pg}}$ in units of $\mathrm{T}$ (upper axis) and $\mathrm{K}$ (lower axis). Also plotted are $\Delta \rho_{c}^{\max }$ vs $T^{*}$ estimated from our analysis of the $\rho_{c}(H, T)$ data in NCCO (open square) [28] and BSCO (open circles) [29]. Dashed line is a $H_{\mathrm{pg}}^{3}$ dependence [Eq. (2)]. Inset: $H_{\mathrm{pg}}$ (at low $T$ ) vs $T^{*}$ (at $0 \mathrm{~T}$ ) in SCCO (square) and BSCCO (circles). Dotted line is the Zeeman scaling line with $g=2$.

simply differs by a parallel shift without any significant differences in the temperature dependencies [inset in Fig. 2(b)], in agreement with a negligible contribution of any thin secondary-phase layers. The peak in $\rho_{c}(H)$ is less pronounced at lower temperatures, and, indeed, at $0.5 \mathrm{~K}$ the peak is barely discerned. Hence, the pseudogap closing field in this configuration is not more than $40 \mathrm{~T}$, giving the $H_{\text {pg }}$ anisotropy less than 1.4. This small anisotropy is in stark contrast with the huge anisotropy of superconducting parameters (e.g., $H_{\text {peak }}$ ) and is in good agreement with the value 1.35 obtained in BSCCO, corresponding to the small anisotropy of the $g$ factor [27]. All this points to the preeminent role of spin correlations over the orbital effects in forming the pseudogap in both BSCCO and SCCO.

Thus, all observed high-field intrinsic features in $n$-type cuprates consistently support a presence of the pseudogap with the origin analogous to the one in $p$-type cuprates. The energy scale $T^{*}$ in the $x \approx 0.14$ SCCO samples is smaller than $T^{*}>300 \mathrm{~K}$ in underdoped BSCCO for $p \sim$ 0.14 [11]. However, if we take into account oxygen deficiency $\delta \sim 0.04-0.045[2,31]$, then electron concentration should be counted as $n=x+2 \delta \sim 0.22-0.23$, corresponding to a strongly overdoped regime with $T^{*}<100 \mathrm{~K}$. Hence, the smallness of the pseudogap energy scale in SCCO may be due to its "overdoped" nature, which may also explain the absence of transition broadening under magnetic fields [16]. This suggests that the electron-hole asymmetry of the pseudogap scale may not be as severe as in prior views.
We thank B. Brandt for the technical assistance at NHMFL, supported by NSF. This work was partly supported by 21st Century COE Program No. 14213201, and a Grant-in-Aid for Scientific Research from MEXT. T. K. was supported by JSPS.

*Electronic address: shibauchi@mailaps.org

[1] Y. Tokura, H. Takagi, and S. Uchida, Nature (London) 337, 345 (1989).

[2] H. Takagi, S. Uchida, and Y. Tokura, Phys. Rev. Lett. 62, 1197 (1989).

[3] J. Orenstein and A. Millis, Science 288, 468 (2000); T. Timusk and B. Statt, Rep. Prog. Phys. 62, 61 (1999).

[4] Y. Onose et al., Phys. Rev. Lett. 87, 217001 (2001); Phys. Rev. B 69, 024504 (2004).

[5] N.P. Armitage et al., Phys. Rev. Lett. 88, 257001 (2002).

[6] N.P. Armitage et al., Phys. Rev. Lett. 87, 147003 (2001).

[7] A. Koitzsch et al., Phys. Rev. B 67, 184522 (2003).

[8] L. Alff et al., Nature (London) 422, 698 (2003).

[9] G.-q. Zheng et al., Phys. Rev. Lett. 90, 197005 (2003).

[10] R. W. Hill et al., Nature (London) 414, 711 (2001).

[11] T. Shibauchi et al., Phys. Rev. Lett. 86, 5763 (2001).

[12] Y.F. Yan et al., Phys. Rev. B 52, R751 (1995).

[13] N. Morozov et al., Phys. Rev. Lett. 84, 1784 (2000).

[14] P. K. Mang et al., Phys. Rev. B 70, 094507 (2004).

[15] Y. Dalichaouch et al., Phys. Rev. Lett. 64, 599 (1990).

[16] Y. Hidaka and M. Suzuki, Nature (London) 338, 635 (1989).

[17] J.L. Peng, Z. Y. Li, and R.L. Greene, Physica (Amsterdam) 177C, 79 (1991).

[18] R. Liang et al., Physica (Amsterdam) 195C, 51 (1992).

[19] K. Tanabeet al., Phys. Rev. B 53, 9348 (1996).

[20] Y. Hidaka et al., J. Phys. Soc. Jpn. 60, 1185 (1991).

[21] This problem is possibly avoided in thin films [see P. Fournier et al., Phys. Rev. Lett. 81, 4720 (1998); T. Sekitani et al., J. Phys. Chem. Solids 63, 1089 (2002); Y. Dagan et al., Phys. Rev. Lett. 92, 167001 (2004)], although there a substrate lattice mismatch (and the associated strain) can modify the charge transport and $T_{c}$.

[22] In BSCCO, large negative MR corresponding to a larger pseudogap energy scale overwhelms the positive $H^{2}$ term.

[23] T. Shibauchi et al., Phys. Rev. B 67, 064514 (2003).

[24] K. Schlenga et al., Physica (Amsterdam) 235-240C, 3273 (1994).

[25] The $45 \mathrm{~T}$ hybrid dc magnet at NHMFL comprises $11.5 \mathrm{~T}$ superconducting and $33.5 \mathrm{~T}$ resistive magnets. A capacitance sensor was used for temperature control.

[26] At higher $T, \alpha$ becomes smaller as the intrinsic $\rho_{c}$ increases, consistent with Kohler's rule.

[27] L. Krusin-Elbaum, T. Shibauchi, and C. H. Mielke, Phys. Rev. Lett. 92, 097005 (2004).

[28] H. J. Kang et al., Nature (London) 423, 522 (2003).

[29] Y. Ando et al., Phys. Rev. Lett. 77, 2065 (1996); 79, 2595(E) (1997); S. Ono et al., Phys. Rev. B 70, 224521 (2004); To extract $T^{*}$, we focus on the relatively high temperature $\rho_{c}(T)$ data to avoid disorder issues in BSCO.

[30] T. Kimura et al., Phys. Rev. B 53, 8733 (1996).

[31] J.S. Kim and D. R. Gaskell, Physica (Amsterdam) 209C, 381 (1993). 